\title{
Shuttle interference effects in the rat depend upon activity during prior shock: A replication
}

\author{
CHARLES R. CROWELL and D. CHRIS ANDERSON \\ University of Notre Dame, Notre Dame, Indiana 46556
}

\begin{abstract}
An omission-training procedure involving a passive-escape contingency was used to reduce the amount of movement during shock exhibited by rats below that of yoked controls exposed to a physically identical but inescapable shock. Subsequent tests for interference with two-way, FR 1 escape/avoidance learning revealed only the passive-escape subjects to be impaired relative to previously restrained but nonshocked controls. These findings duplicated the results of an experiment by Anderson et al. and were viewed as indicating that behavior during shock, rather than uncontrollability, is the critical determinant of proactive shock treatment effects.
\end{abstract}

In the first of two experiments Anderson, Crowell, Cunningham, and Lupo (1979) demonstrated that omission training in the form of a passive-escape procedure could be used with rats to reduce levels of movement during shock below those exhibited by control subjects exposed to inescapable shock. The general design of this study was similar to that employed by Maier (1970) with dogs, in which one group received explicit negative reinforcement for not displacing panel manipulanda adjacent to the head, while a second group, comprising subjects yoked to those in the first, experienced physically and temporally identical exposure to uncontrollable shock. Unlike Maier, however, Anderson et al. employed an ac shock stimulus that was rapidly interrupted (i.e., pulsating) rather than temporally continuous. This difference may be important in light of findings by Lawry, Lupo, Overmier, Kochevar, Hollis, and Anderson (1978) that pulsating and continuous forms of ac shock tend to promote different levels of intrashock movement in dogs and rats, even without the use of any special training procedures. Over the course of a series of 5-sec inescapable ac shocks, Lawry et al. observed that a pulsating form produced relatively sustained activity during shock. On the other hand, a continuous form was accompanied by a significantly lower level of intrashock movement that was comparable to what might be observed using a passive-escape procedure. Thus, it seems likely that the inherent movement-producing properties of the shock stimuli employed by Anderson et al. and Maier were not the same. And unfortunately, as noted below, the common purpose of these studies may not have been equally served by their respective temporal forms of shock.

In essence, both the Anderson et al. (1979) and

Thanks are extended to J. Madden, J. Rollins, and J. V. Lupo for assistance in various phases of this research and to $C$. $L$. Cunningham and J. S. Brown for helpful suggestions in the preparation of this paper. Requests for reprints should be addressed to either author, Department of Psychology, University of Notre Dame, Notre Dame, Indiana 46556.
Maier (1970) experiments were attempts to assess the validity of opposing views of the phenomenon of interference with escape/avoidance learning that has been reported to follow a treatment consisting of exposure to inescapable shock (e.g., Overmier \& Seligman, 1967). According to one theory, the uncontrollable nature of the treatment shock leads an organism to develop the belief that its behavior has no effect upon shock (e.g., Maier \& Seligman, 1976). Such a "cognitive set," in turn, allegedly carries over to the escape/avoidance task, where it results in substantial reduction of the likelihood that organisms will acquire the appropriate instrumental response. In contrast, according to another view of interference, covert contingencies supplied by treatment shock may favor the occurrence of immobility reactions over other responses during shock (e.g., Bracewell \& Black, 1974). Consequently, subjects are thought to acquire tendencies to remain motionless during shock that may generalize to the cues of a subsequent escape/avoidance test situation wherein they prove incompatible with responding normally observed there. In an effort to differentiate these alternative conceptions, both Anderson et al. and Maier attempted to "pit" the factor of immobility during shock against that of shock inescapability using a passive-escape procedure to provide one group with control over shock while explicitly reinforcing intrashock immobility. Another group was yoked to the first and thereby received comparable shock exposure but independent of its activity level. If interference resulted chiefly from inactivity learned during shock, then the passive-escape condition should have produced the greater subsequent impairment of test performance relative to a group not given shock treatment. Alternatively, if uncontrollability of shock was the critical factor, then the reverse pattern of results should have occurred.

Obviously, the logic of these expectations is correct only if the yoked shock treatment, supposedly involving inescapable shock, was in fact accompanied by 
significantly higher levels of movement during shock than were those produced by the omission training procedure. However, in view of the results of the Lawry et al. (1978) study, there could be no assurance of this outcome with a continuous shock, since, as noted above, it seems closely to resemble a passive-escape procedure in promoting relatively low levels of movement during shock. On the other hand, a difference in terminal movement between groups in the appropriate direction might be more likely with a pulsating form of ac shock, since, at least under some circumstances, inescapable presentations of this stimulus are known to have movement-sustaining properties (Lawry et al., 1978).

For these reasons, Anderson et al. (1979) employed a pulsating rather than a continuous form of ac shock. Their findings indicated that passive-escape subjects moved significantly less than yoked rats during final treatment shock presentations and also exhibited reliably more impairment during subsequent escape/avoidance training. In contrast, Maier (1970) observed his yoked subjects to show the greater impairment during testing. But, since a direct comparison of movement during shock was not made for passive-escape and yoked groups in the latter study, it is impossible to discern whether Maier's results actually were discordant with the relative intrashock activity levels promoted by his treatments. That is, if for some reason the continuous ac shock employed by Maier actually resulted in more complete immobility when it was inescapable (i.e., in the yoked condition) than when it was used in conjunction with omission training, then the observed results would not be inconsistent with a competing-response account. Thus, in the absence of relevant data, it must be considered possible that the dissimilar outcomes of the Anderson et al. and Maier studies can be attributed entirely to the different temporal forms of shock used in each case rather than to species or other methodological factors.

As a first step toward validation of this possibility, it seems desirable to replicate the procedures of Anderson et al. (1979) with rats in an effort to show that the previous findings were not the spurious consequence of sampling error. Accordingly, in the present study, the same procedures were employed as in Anderson et al. (1979, Experiment 1) except that only 3 , rather than 8, days of escape/âvoidance testing were used.

\section{METHOD}

\section{Subjects}

Forty-eight naive male albino Sprague-Dawley rats 90-100 days of age, were used. They were housed in separate cages on an ad-lib food and water diet. All were handled and weighed daily, and all were acclimated 7-10 days in the laboratory prior to experimentation.

\section{Apparatus}

Shock treatment. A flat acrylic platform, supported on three wooden dowels over a plywood base, was used for restraining subjects (modified after Lykken \& Rose, 1959). The platform included separate relief areas for the subject's genitals, head, and limbs. One dowel was located between the forelimb holes, and one dowel was located behind each hindlimb hole.
The platform was contained in a sound- and light-resistant ventilated refrigerator shell.

Two head panels were fitted to the restraint platform. Each panel was $7.62 \mathrm{~cm}$ long $\times 5.1 \mathrm{~cm}$ wide and was located with the shorter side perpendicular to the platform approximately .65$.70 \mathrm{~cm}$ from either side of the rat's head. They formed a V shape that conformed roughly to the curvature of the animal's head. Microswitches were activated when these panels were pressed (15 g pressure). These panels provided a means of recording head and upper body movement during treatment shock.

The treatment shock source was the output of a Lafayette ac shock generator (Model 5225) wired in series with a sequence of resistors through a stepping switch. The resistors were arranged so that successive operations of the stepping switch would increase the nominal current level in .3-mA steps to a maximum nominal intensity of $1.5 \mathrm{~mA}$. This shock was interrupted 10 times per second with a Lehigh Valley pulse-stream generator (Model 1670), which produced square-wave on-off times of $.05 \mathrm{sec}$. All treatment contingencies were controlled with solid state equipment and a laboratory computer housed in another room.

Testing. An $11.3 \mathrm{~cm}$ wide $\times 59.7 \mathrm{~cm}$ long $\times 25.0 \mathrm{~cm}$ high (inside) shuttlebox was used. The shuttlebox contained a grid floor $(.32-\mathrm{cm}$-diam stainless steel rods mounted $1.27 \mathrm{~cm}$ apart), stainless steel walls, a cylindrical hurdle $(5.1 \mathrm{~cm}$ in diameter), and a $7.5-\mathrm{W}$ lamp that served as the CS, located $22 \mathrm{~cm}$ above the floor at each end of the chamber. The hurdle extended $6.3 \mathrm{~cm}$ above the floor and was mounted on ball bearings, thereby preventing the subject from perching on it. The grid floor was divided in half, and microswitches were attached to each floor assembly. A full crossing from one compartment to the other was required in order to activate the switches and define a response. The test shock was the scrambled $40-\mathrm{V}$ ac output of a transformer wired in series with a 22-kohm resistor. The walls and hurdle were connected to the grid-shock circuit. The computer recorded response latencies and intertrial crossings and controlled all stimulus presentations.

\section{Procedure}

The experiment was conducted in 12 replications of four rats, one in each of the four groups. The rats were ranked by weight for each replication, and animals in each block of four subjects were randomly assigned to one of four groups. Two groups were restrained and exposed to shock, either in connection with the passive-escape or the yoked treatment conditions. One nonshocked control group was simply restrained for a comparable period of time (restrained group), whereas the other nonshocked control was unrestrained (unrestrained group).

On each treatment day, each restrained subject was lightly anesthetized (20- to 40 -sec exposure to ether in a closed jar) and strapped onto the acrylic platform by wrapping a 2 -in.-wide elastic bandage around the torso and by taping the forelimbs to the front dowel and the hindlimbs to the appropriate rear dowels. A polished copper electrode, lightly moistened with electrode paste, was firmly attached to the outstretched sole of each hind paw with adhesive tape. Treatment began approximately $10 \mathrm{~min}$ after etherization and $4 \mathrm{~min}$ after placement of the rat in the refrigerator shell.

Rats in the shock-treated passive-escapable group (ST-PE) could minimize both the duration and the intensity of each shock by learning to remain relatively still, thereby not pressing the head panels during shock for a predetermined period of time. On the first trial of the session, the duration of nonresponding required to minimize shock parameters successfully (referred to hereafter as the "criterion duration") was 2 sec. If, at any time within $15 \mathrm{sec}$ of shock onset, the rat remained "motionless" (i.e., did not displace a head panel) for an interval of time equal to the criterion duration, shock terminated and the trial was scored as "correct." If the rat did not attain the criterion within $15 \mathrm{sec}$, the trial terminated automatically and was scored as "incorrect." This procedure was repeated on 
each succeeding trial with the same criterion value until the rat showed evidence of mastery at that duration, at which point the criterion was increased slightly toward the ultimate goal of a full $15 \mathrm{sec}$. Specifically, in any one of the possible $30 \mathrm{con}-$ secutive blocks of five trials beginning with Trial 1 , three correct trials in a row or four out of five correct would result in a 1 -sec increase in the criterion duration for the next block of trials. If the rat made seven consecutive "errors" at any criterion duration, however, the criterion value was decreased by $1 \mathrm{sec}$ for the next block.

As noted, these rats could also prevent programmed increases in shock intensity by not hitting the head panels during shock. Each shock was presented at the lowest intensity $(.3 \mathrm{~mA})$, but each of the first four panel presses increased intensity by $.3 \mathrm{~mA}(1.5 \mathrm{~mA}$ maximum). Shocks were delivered at intervals averaging $60 \mathrm{sec}( \pm 15 \mathrm{sec})$ until the rat achieved the learning criterion at the $15-\mathrm{sec}$ level or until it had received a total of 150 shocks. Number of panel displacements was recorded both during and between shock presentations.

The subjects of the shock-treated yoked group (ST-Y) were individually paired with subjects of the passive-escapable group, and they thus received the same number, pattern, and total amount of shock. Number of panel displacements also was recorded for this group. Rats in the restrained group (R) were simply strapped to the platform in the sound-deadened enclosure for periods that were comparable to those of shock-treated subjects. The rats in the unrestrained group (U) were placed in individual carrying cages for the same periods of time.

Testing. All subjects were given shuttle escape-avoidance training $24 \mathrm{~h}$ following the shock-treatment day. After a 2-min adaptation period to the apparatus, 12 trials were given with a CS-US (light-shock) interval of $5 \mathrm{sec}$ and a variable intertrial interval of $60 \mathrm{sec}( \pm 30 \mathrm{sec})$. Trials terminated automatically after $60 \mathrm{sec}$ if the rat failed to respond. This procedure was repeated on each of the next 2 days, for a total of 3 test days. Latency to respond after CS onset and number of avoidance responses were recorded.

\section{RESULTS}

\section{Shock Treatment}

All of the subjects of Group ST-PE both reached and successfully mastered the criterion of not hitting a head panel during $15 \mathrm{sec}$ of shock within 150 shock presentations. The mean number of trials to reach the $15-\mathrm{sec}$ criterion level was 92.1. Three subjects met the $15-\mathrm{sec}$ criterion level within the minimum number (i.e., 70) of trials possible. Six subjects required one decrease in criterion duration, and one subject required two decrements (consecutive) before the 15-sec maximum was achieved. The mean criterion duration achieved before the initial reversal (decrement) occurred for the seven passive-escapable subjects was $11.1 \mathrm{sec}$.

The mean number of intershock movements for each half of the treatment session was obtained for each subject and was converted to movements per second. Movements per second during shock were determined for each subject separately for each shock criterion level. Mean intershock movements per second declined over session halves $[F(1,22)=43.2, p<.01]$ equally for both shock-treated groups. Mean movement rate during shocks also markedly declined over the shock criterion durations, but not equally for the two shock groups. The latter finding is illustrated in Figure 1, in which it can be noted that the mean movements per second for

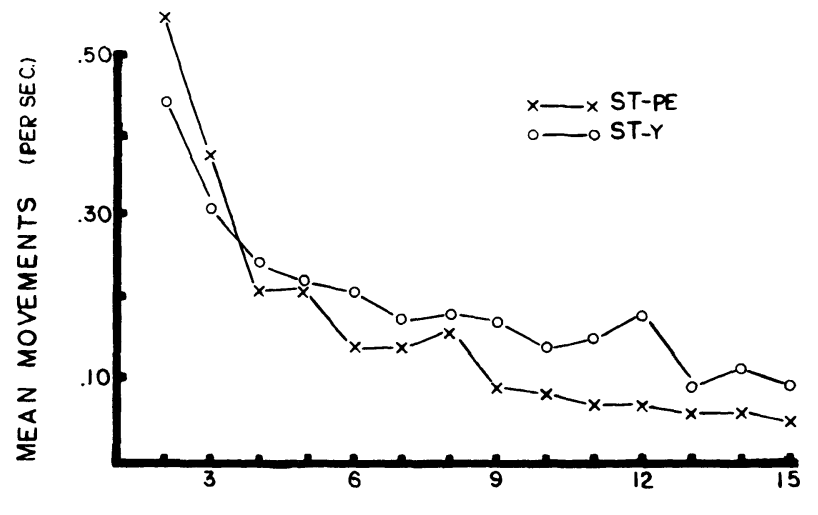

CRITERION SHOCK DURATION (IN SEC.)

Figure 1. Mean movements per second during treatment shock as a function of criterion duration for groups exposed to a passiveescape procedure (ST-PE) or a yoked, inescapable shock (ST-Y).

Group ST-PE, although arithmetically higher than those for Group ST.Y at the outset of shock treatment, were appreciably lower by the end of the treatment session.

These observations regarding the movements/second data during shock were supported statistically by the results of a 2 (groups) by 14 (criterion levels) ANOVA. The only significant effects in this analysis were due to criterion levels $[\mathrm{F}(13,386)=36.7, \mathrm{p}<.01]$ and the interaction of groups with criterion levels $[\mathrm{F}(13,386)=$ 2.22, $\mathrm{p}<.01]$. Newman-Keuls $(\alpha=.05)$ follow-up comparisons of the groups effect at each criterion level indicated that reliable group differences did not occur until the $11-\mathrm{sec}$ criterion level, and they were significant at each level thereafter except at $13 \mathrm{sec}$.

\section{Testing}

A median latency to respond from CS onset was determined for each subject over the 12 trials on each day. Mean-median latencies for each group as a function of test days are depicted in Figure 2. It is apparent from this graph that Group ST-PE showed longer response latencies than did Group ST-Y, and both shock-treated groups generally were slower to escape than were either of the control groups. Further, all four groups showed a decrease in mean latencies over the 3 test days. An overall 4 (groups) by 3 (days) ANOVA yielded significant effects for groups $[F(3,44)=5.18, p<.005]$, days $[\mathrm{F}(2,88)=12.16, \mathrm{p}<.001]$, and Groups by Days $[F(6,88)=2.22, \quad p<.05]$. The latter interaction prompted separate evaluation of differences among groups at each day. Newman-Keuls multiple comparisons $(\alpha=.05)$ indicated that Group $R$ differed significantly from Group U only on Days 1 and 2. Also, Group ST-PE was significantly different from Group ST-Y on Days 1 and 2. In view of the apparent restraint effect among the control groups with this measure, only Group $R$ was used for the purpose of assessing interference effects. Dunnett comparisons $(\alpha=.05)$ of each shock-treated group with Group $R$ 


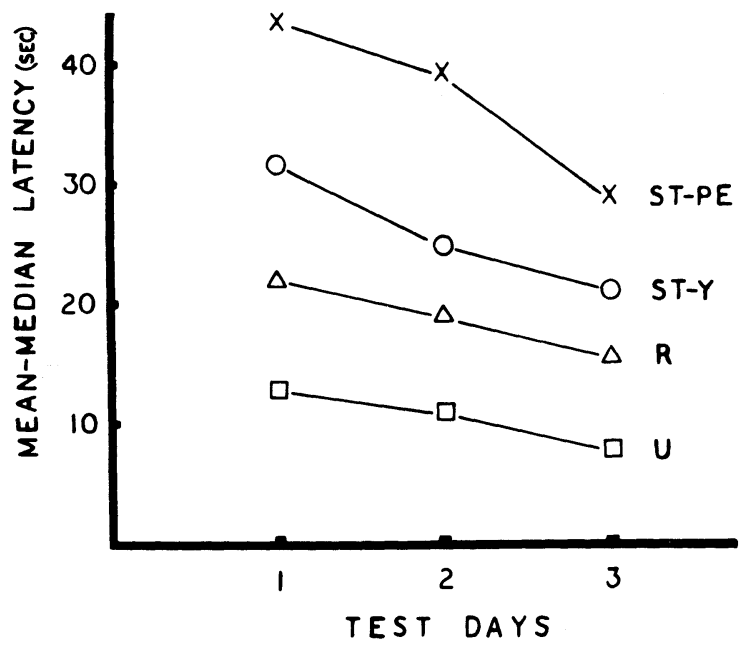

Figure 2. Mean-median escape/avoidance response latency from CS onset by days for groups previously exposed either to

revealed that the impairment exhibited by Group ST-PE was reliable on all days. Group ST.Y, however, did not differ significantly from Group R on any day.

All but Group ST-PE showed an increase in number of avoidance responses over the 3 test days. Averaged over days for each group, the mean numbers of avoidances were $1.20,1.73$, and 2.66 for Groups $R, U$, and ST-Y, respectively. In contrast, Group ST-PE averaged only .41 avoidance responses over days. A 4 (groups) by 3 (days) ANOVA of these measures produced significant effects only for groups $[\mathrm{F}(3,44)=4.35, \mathrm{p}<.01]$ and days $[F(2,88)=3.25, p<.05]$. Since the difference between Groups $R$ and $U$ was not reliable with this measure, all possible pairwise comparisons among groups (Newman-Keuls, $\alpha=.05$ ) were used as both a follow-up of the overall groups effect and a means of assessing interference. These tests indicated that the overall groups effect resulted primarily from significant differences between Group ST-PE and all others. No other comparisons were reliable with this measure.

\section{DISCUSSION}

The effects of the passive-escape procedure on intrashock activity and escape/avoidance learning in the present study were virtually identical to those reported by Anderson et al. (1979, Experiment 1). In both instances, omission training was found to result by the end of treatment in significantly lower levels of movement during shock than those promoted by exposure to a comparable but inescapable shock. During testing, passiveescape subjects in both studies were found to exhibit greater impairment of escape/avoidance learning than yoked controls, relative to the performance of subjects not previously shocked.

The yoked, inescapable shock treatments in both studies also produced comparable decrements in within-shock activity over the course of treatment, although, as noted, these decreases were smaller than those resulting from omission training. However, only in the Anderson et al. (1979, Experiment 1) study did this shock treatment result in a significant interference effect, and then only on the first 2 test days. By Day 3 of testing, and on each of the remaining 5 days in that experiment, the test performance of yoked rats was indistinguishable from that of previously restrained but nonshocked controls. In the present study, a similar pattern of results was obtained in terms of differences between group means over the 3 test days, but on no day did the difference prove reliable.

Thus, even though it promoted a substantial reduction over the course of treatment in movement during shock in both studies, the yoked, inescapable shock treatment produced at most in either case only an initial disruption of test performance. As noted by Anderson et al. (1979), such findings may be viewed as an indication that the mere fact of a reduction in activity during shock is not a sufficient condition for the later occurrence of impairment. Indeed, this outcome could be considered consistent with a competing-response account, since, according to such a view, reductions in intrashock movement would be a reflection of an associative process only when conditions were favorable for the learning of immobility during shock cues. Of the two shock treatments in the present study, only the one involving explicit reinforcement for reduced movement during shock would seem to provide such circumstances.

The present results serve mainly to confirm the reliability of the effects reported by Anderson et al. (1979, Experiment 1). In addition, they tend further to support the view that behavior during shock, rather than shock uncontrollability, is the critical determinant of proactive interference effects. If Maier's (1970) disparate findings are to be reconciled with this view, the next step would be to show that the present procedures, when used with the shock stimulus employed by Maier, produce a pattern of results that is opposite the above, both in terms of activity during shock and in terms of interference. However, according to competing-response theory, such a result should occur only if a yoked, inescapable shock treatment can produce greater learned inactivity to shock than that resulting from a passive-escape procedure when a continuous ac shock is employed. Although an argument to this effect has been advanced, for reasons noted by Anderson et al. (1979), further research will be required to verify this possibility.

\section{REFERENCES}

Anderson, D. C., Crowell, C. R., Cunningham, C. L., \& LuPo, J. V. Behavior during shock exposure as a determinant of subsequent interference with subsequent escape-avoidance learning in the rat. Journal of Experimental Psychology: Animal Behavior Processes, 1979, 5, 243-257.

Bracewell, R. J., \& Black, A. H. The effects of restraint and noncontingent preshock on subsequent escape learning in the rat. Learning and Motivation, 1974, 5, 53-69.

Lawry, J. A., Lupo, V., Overmier, J. J., Kochevar, J., Hollis, K. L., \& ANDERSon, D. C. Interference with avoidance behavior as a function of qualitative properties of inescapable shocks. Animal Learning \& Behavior, 1978, 6, 147-154.

LyкKen, D. T., \& Rose, R. A rat-holder with electrodes for GSR measurement. American Journal of Psychology, 1959, 72, 621-622.

MaIER, S. F. Failure to escape traumatic shock. Learning and Motivation, 1970, 1, 157-169.

Maier, S. F., \& Seligman, M. E. P. Learned helplessness: Theory and evidence. Journal of Experimental Psychology: General, 1976, 105, 3-46.

Overmier, J. B., \& Seligman, M. E. P. Effects of inescapable shock upon subsequent escape and avoidance responding. Journal of Comparative and Physiological Psychology, 1967, 63, 28-33.

(Received for publication September 21, 1979.) 